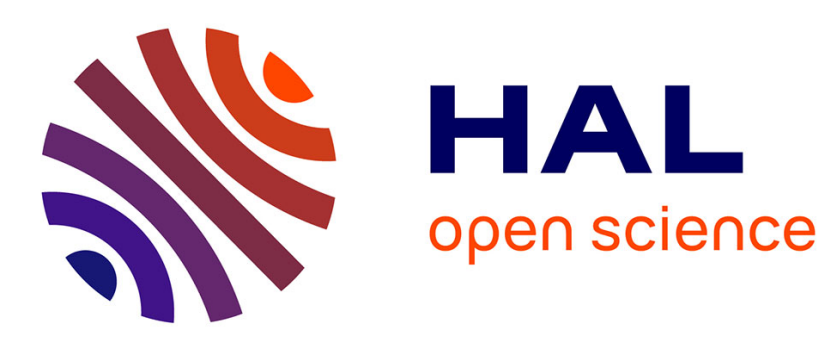

\title{
Time-domain near-field/near-field transform with PWS operations
}

B. Ravelo, Yehan Liu, J. Ben Hadj Slama

\section{To cite this version:}

B. Ravelo, Yehan Liu, J. Ben Hadj Slama. Time-domain near-field/near-field transform with PWS operations. European Physical Journal: Applied Physics, 2011, 53 (3), 10.1051/epjap/2011100447. hal-00672784

\section{HAL Id: hal-00672784 \\ https://hal.science/hal-00672784}

Submitted on 22 Feb 2012

HAL is a multi-disciplinary open access archive for the deposit and dissemination of scientific research documents, whether they are published or not. The documents may come from teaching and research institutions in France or abroad, or from public or private research centers.
L'archive ouverte pluridisciplinaire HAL, est destinée au dépôt et à la diffusion de documents scientifiques de niveau recherche, publiés ou non, émanant des établissements d'enseignement et de recherche français ou étrangers, des laboratoires publics ou privés. 


\title{
Time-domain near-field/near-field transform with PWS operations
}

\author{
B. Ravelo ${ }^{1}$, Y. Liu ${ }^{1}$ and J. B. H. Slama ${ }^{2}$ \\ ${ }^{1}$ IRSEEM, EA 4353, \\ Engineering School ESIGELEC, \\ Technopole du Madrillet, Avenue Galilée, BP10024, \\ 76801 Saint Etienne du Rouvray Cedex, France. \\ ${ }^{2}$ LSE, National Institute of Applied Sciences and Technology, \\ BP 676, 1080 Tunis Cedex, Tunisia. \\ *blaise.ravelo@yahoo.fr (the corresponding author), \\ yang.liu@esigelec.fr, \\ bhslama@yahoo.fr.
}

\begin{abstract}
This article deals with the development of computation method dedicated to the extraction of the transient EM-near-field at certain distance from the given 2-D data for the baseband application up to GHz. As described in the methodological analysis, it is based on the use of fft combined with the plane wave spectrum (PWS) operation. In order to verify the efficiency of the introduced method, a radiating source formed by the combination of electric dipoles excited by a short duration transient pulse current with a spectrum bandwidth of about $5 \mathrm{GHz}$ is considered. It was shown that compared to the direct calculation, one gets the same behaviors of magnetic near-field components $H_{x}, H_{y}$ and $H_{z}$ with the presented extraction method, in the planes placed at $\{3 \mathrm{~mm}, 8 \mathrm{~mm}, 13 \mathrm{~mm}\}$ of the initial reference plane. To confirm the relevance of the proposed transform, validation with a standard commercial tool was performed. In future, we envisage to exploit the proposed computation method to predict the transient electromagnetic (EM) field emissions notably in the microwave electronic devices for the EMC applications.
\end{abstract}

Keywords- EMC application, Near-field, Planar transform, Plane wave spectrum (PWS), Spacetime analysis, Time-domain computation, Transient propagation.

\section{INTRODUCTION}

According to the exponential growth of electronic system integration density in the confined space for the automotive, automatic and aeronautronic industrial environments, the electromagnetic interference (EMI) characterization becomes currently more and more important [1-7]. Therefore, this disturbing phenomenon should be taken into account by engineer designers in particular during the study of RF-/digital- and powerelectronic components for attempting to reduce the EM disturbing source. Due to the increase of the operating 
frequency which attains nowadays several $\mathrm{GHz}$ and the simultaneous commutations of several millions of electronic logic gates in the integrated circuits (IC), undesired current peaks can be created in certain parts of the integrated system. This undesired pulse current peak can be critical for RF electronic devices because of the eventual interferences with other communication systems working at the same frequency. For this typical pulse phenomenon, the mean energy of these seemingly transient or non-frequent peaks is almost negligible in frequency domain. However, the instantaneous energy providing these transient phenomena can be significant in the time-domain [8-16]. That is why the analysis of the physical and electrical effects in the EM environment owing to the different electronic components and systems must be mastered. In this way, most of the traditional existing modeling and characterization methods for near- and far-field characterization techniques were carried out in the frequency domain [17-20]. More generally, the transient EM field propagation is one of concern of mathematicians and physicists of different scientist disciplines [21-24]. The assessment and the characterization of EM-field emissions in 3-D near-zone for the given radiating electric- or electronic- devices in time domain remains complicated in particular if the phenomenon appears briefly.

For this reason, we are motivated to conduct the present research work. It is aimed at the development of spacetime computation technique enabling to determine the EM near-field cartography in the plan arbitrarily placed at a certain distance of the given 2-D EM data in the time-domain. To do this, one employs the space-frequency transform theory known as the plan wave spectrum (PWS) method. As introduced in [25-32], this spacetransform is exactly similar to the fast Fourier transform in two-dimension domain.

For the better comprehension, this article is structured as follows. Section 2 is consecrated to the theoretical aspect and the methodological description of the under study technique. In Section 3 , an example of application dedicated to the calculation of the three magnetic field components $H_{x}, H_{y}$ and $H_{z}$ in the rectangular plane placed at different levels $\{3 \mathrm{~mm}, 8 \mathrm{~mm}, 13 \mathrm{~mm}\}$ from the given data is offered by considering an arbitrary combination of five electric dipoles excited by transient pulse electric current. The last section describes the conclusion and the prospects of this work.

\section{DESCRIPTION OF THE PROPOSED TECHNIQUE FOR THE EM NEAR-FIELD TRANSFORM}

Along this study, one supposes that the radiated EM field is recorded at the arbitrary point $M(x, y, z)$ which is referenced with the Cartesian system $(O x y z)$. By taking into account the time variable parameter, the study of the whole EM field propagation can be transposed to the analysis of a typically four-dimension tensorial matrix. To do this, one considers the space-time tensorial data of the EM field components expressed as $\vec{X}(x, y, z, t)=[\vec{E}(x, y, z, t), \vec{H}(x, y, z, t)]$. In this Cartesian coordinate system $(O x y z)$, one denotes $X_{x}(x, y, z, t)$, $X_{y}(x, y, z, t)$ and $X_{z}(x, y, z, t)$, the three components of $X$-field radiated in the rectangular plan delimited by $X_{\min }, X_{\max }$, $Y_{\min }$ and $Y_{\max }$ as represented in Fig. 1 for the time-vector $t=\left[0, T_{\max }\right]$ with time step $\Delta t$. According to the signal processing theory, the corresponding frequency evolution of the field-vector $\vec{X}$ can be determined toward the fast Fourier transform $(f f t)$ in the equivalent frequency range varying from $f_{\text {min }}=f_{\text {step }}=1 / T_{\text {max }}$ to $f_{\text {max }}=1 / \Delta t$ through the following classical relation:

$$
\vec{X}(x, y, z, f)=\int_{-\infty}^{\infty} \vec{X}(x, y, z, t) e^{-j 2 \pi f t} d t,
$$

where $j$ is the complex number $\sqrt{-1}$. 

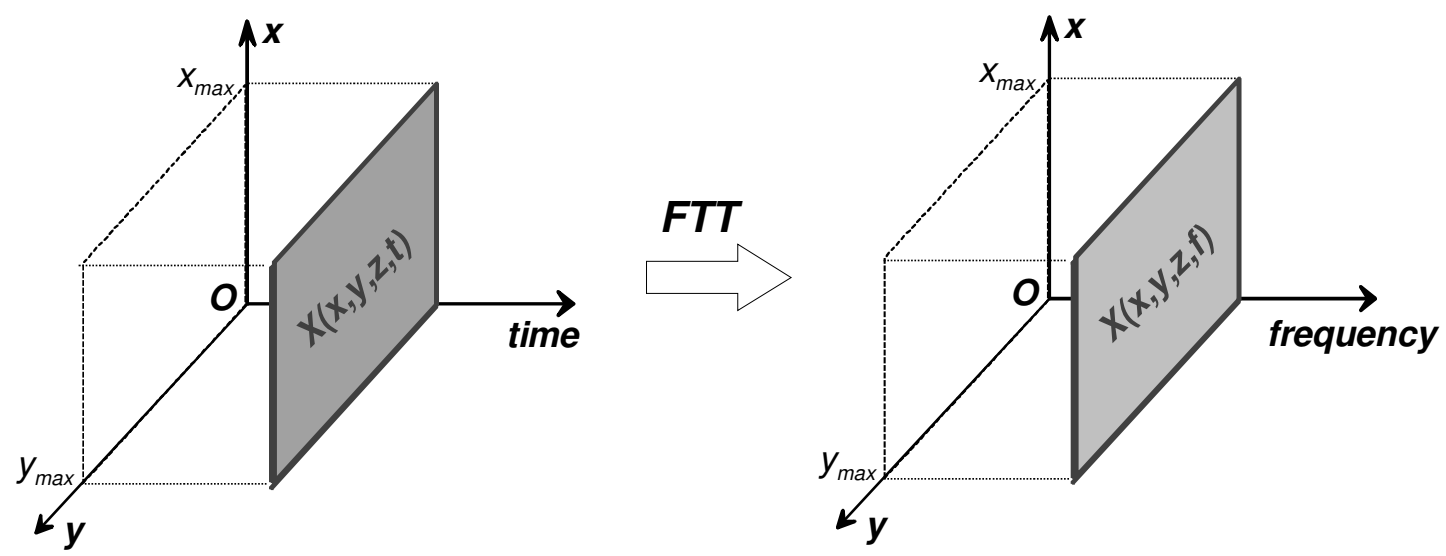

Fig. 1: Illustration of the calculation method by using the space-time data $\vec{X}(x, y, z, t)$ and the corresponding space-frequency data $\vec{X}(x, y, z, f)$ obtained with the FFT operation.

To calculate the radiated $\vec{X}$-field in the other plane $z_{0}$-distant from the reference plane where the given data is mapped, we proceed with the plane wave spectrum (PWS) method as illustrated in Fig. 2.
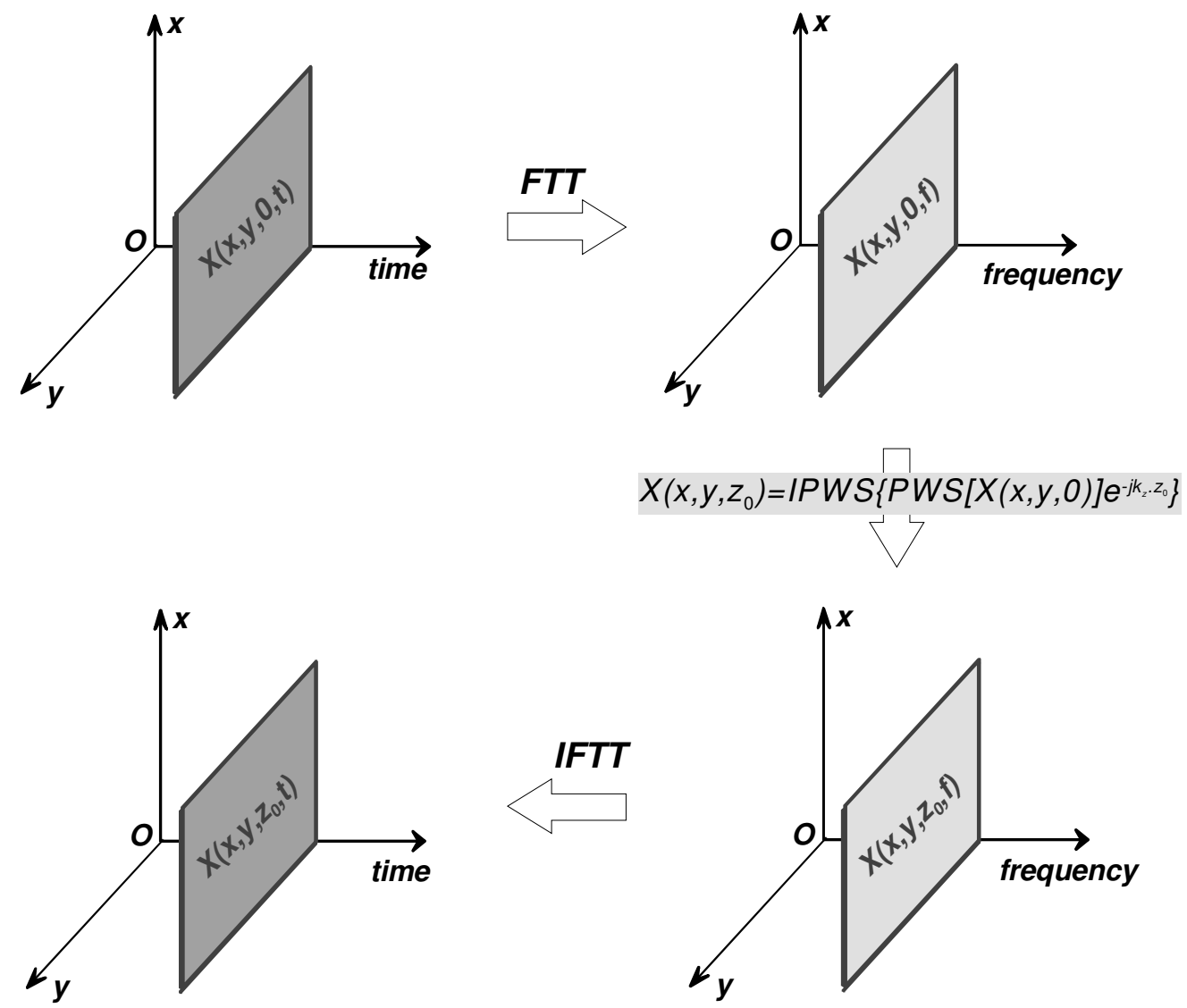

Fig. 2: Different steps for the extraction of $\vec{X}\left(x, y, z_{0}, t\right)$ from $\vec{X}(x, y, 0, t)$. 
In summary, the space-time data $\vec{X}(x, y, 0, t)$ is first, converted in space-frequency data $\vec{X}(x, y, 0, f)$. Then, with the obtained data in frequency domain, one can apply the PWS operation by assuming $\vec{k}(f)$ the wave vector associated to each frequency, $f$ and referenced in the classical Cartesian coordinate system (Oxyz) having the unit vectors $\overrightarrow{u_{x}}, \overrightarrow{u_{y}}$ and $\overrightarrow{u_{z}}$ :

$$
\vec{k}(f)=k_{x}(f) \overrightarrow{u_{x}}+k_{y}(f) \overrightarrow{u_{y}}+k_{z}(f) \overrightarrow{u_{z}} .
$$

One recalls that the modulus of this wave vector which is known also as the wave number at the frequency $f$ is given by:

$$
k(f)=\sqrt{k_{x}^{2}(f)+k_{y}^{2}(f)+k_{z}^{2}(f)}=\frac{2 \pi}{\lambda(f)} .
$$

By denoting $c$ the wave speed in the considered medium, one denotes $\lambda(f)=c / f$ the propagating wavelength at the frequency, $f$. The PWS operation consists in the transformation of the planar EM-field in its 2-D spectrum similar to the fast Fourier transform known with the instruction fft2 in Matlab language. It is mathematically defined by the following expression [25-32]:

$$
\vec{P}_{X(z=0)}\left(k_{x}, k_{y}, f\right)=\frac{1}{4 \pi^{2}} \int_{-\infty}^{\infty} \int_{-\infty}^{\infty} \vec{X}_{z=0}(x, y, f) e^{-j\left[k_{x}(f) x+k_{y}(f) y\right]} d x d y .
$$

The introduced technique of the EM near field field extraction which enables to predict the value of the field surrounding the electronic emitting source was already introduced in [29-31] especially in frequency domain. As depicted in Fig. 3, in the PWS domain, $\vec{P}_{X\left(z=z_{0}\right)}$ at the height, $z=z_{0}$ can be extracted from $\vec{P}_{X(z=0)}\left(k_{x}, k_{y}, f\right)$ with the relationship:

$$
\vec{P}_{X\left(z=z_{0}\right)}\left(k_{x}, k_{y}, f\right)=\vec{P}_{X(z=0)}\left(k_{x}, k_{y}, f\right) e^{-j \cdot k_{z}(f) \cdot z_{0}}
$$

Hence, $\vec{X}_{z=z_{0}}(x, y, f)$ can be merely extracted through IPWS, likely to the ifft2-transform:

$$
\vec{X}(x, y, f)=\frac{1}{4 \pi^{2}} \int_{-\infty}^{\infty} \int_{-\infty}^{\infty} \vec{P}_{X}\left(k_{x}, k_{y}, f\right) e^{-j\left(k_{x} x+k_{y} y\right)} d k_{x} d k_{y} .
$$

Fig. 3 explains the different steps of the proposed near-field to near-field transform which require the successive passage in space- and PWS- domains.

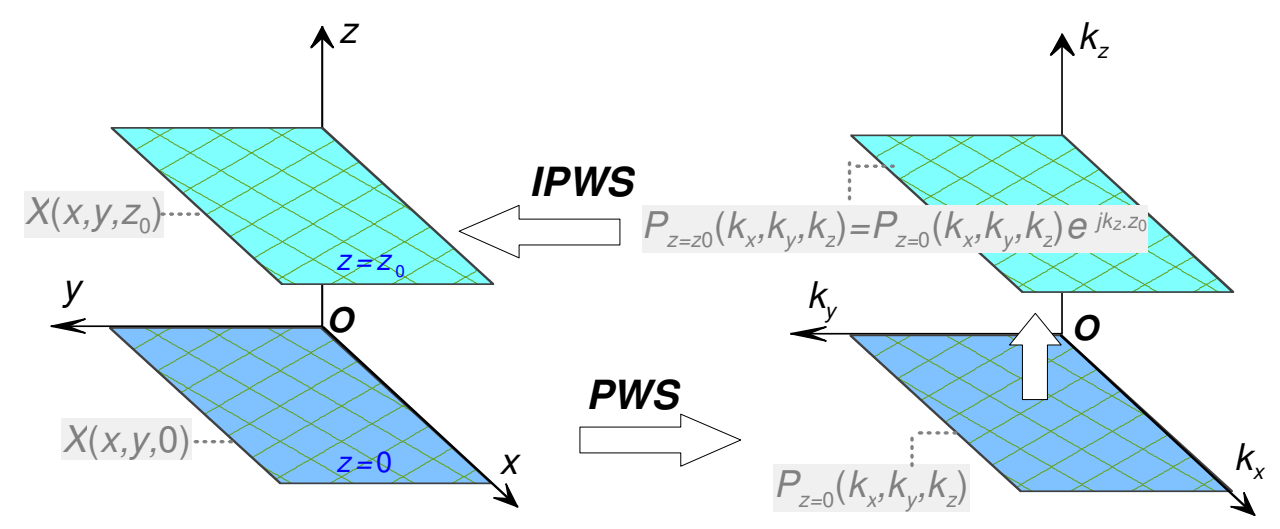

Fig. 3: Calculation principle of frequency data $X\left(x, y, z_{0}\right)$ from $X(x, y, 0)$ by using the PWS transform. 


\section{THEORETICAL RECALL ON THE CONSIDERED EM RADIATION}

As can be seen in Fig. 4, an electric dipole is placed at the origin reference point $O(0,0,0)$ of Cartesian coordinate system $\left(O, \vec{u}_{x}, \vec{u}_{y}, \vec{u}_{z}\right)$ and the arbitrary point $M(x, y, z)$ where the magnetic field will be expressed. This dipole is supposed with physical length, $d$ and excited by time-varying current denoted $i(t)$.

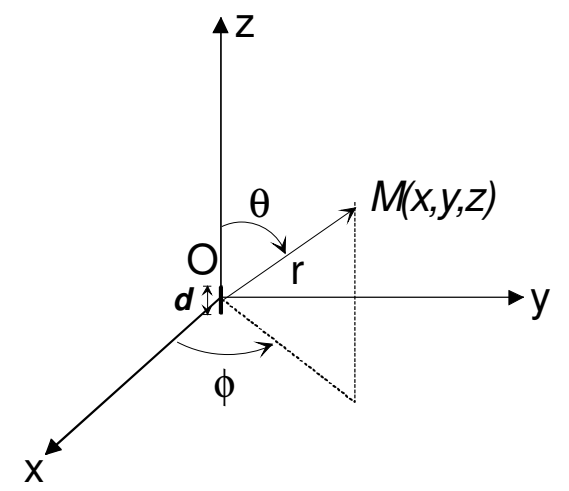

Fig. 4: Elementary electrical dipole placed at the centre of Cartesian coordinate system.

As reported in [13], the expression of dipole moment exhibited by infinitesimal time-varying electric dipole depicted in Fig. 4 is expressed as:

$$
\vec{p}_{E}(r, t)=p(t) \delta(r) \cdot \vec{u}_{z},
$$

where $\delta(r)$ is the Dirac delta function. In this case, the variable moment $p(t)$ is related to the temporal current $i(t)$. By denoting $c$ the wave-velocity in the considered medium, it is clear that the time retarded variable is defined as $\tau=t-r / c$. Hence, the spherical magnetic field components of $\vec{H}=H_{r}(r, t) \vec{u}_{r}+H_{\theta}(r, t) \vec{u}_{\theta}+H_{\varphi}(r, t) \vec{u}_{\varphi}$ are merely given by:

$$
\begin{gathered}
H_{r}(r, t)=0, \\
H_{\theta}(r, t)=0, \\
H_{\varphi}(r, t)=\frac{\sin (\theta)}{2 \pi r^{2}}\left[\frac{\partial p(\tau)}{\partial t}+\frac{r}{c} \frac{\partial^{2} p(\tau)}{\partial t^{2}}\right] .
\end{gathered}
$$

Toward the classical relationship of spherical to rectangular transformation, one can determine the corresponding Cartesian coordinates of these magnetic field components. Then, based-on the time domain fast Fourier transform combined with the PWS transform method, it is possible to generate easily the harmonic components of $\vec{H}$. We will demonstrate later that using the PWS method applied to these frequency or harmonic components, it is possible to extract the $z$-direction component of the radiating structure knowing the two other horizontal ones ( $x$ - and $y$ - components).

\section{COMPUTATION RESULTS}

In this section, the radiating source is assumed as the combination of five electric dipoles, $D_{i}$ with $(i=\{1 \ldots 5\})$ as represented in Fig. 5. These dipoles are placed in the horizontal plane $z=0$ at the points with coordinates 
$D_{i}(R \cos [2(i-1) \pi / 5], R \sin [2(i-1) \pi / 5])$. We emphasize that we choose voluntarily this arbitrary association of EM dipoles because as reported in the recent studies published in [17, 18], from any EM near-field maps radiated by electronic equipments, one can synthesize an optimal set of dipoles placed in the horizontal plane. During the calculation, one chooses arbitrarily $R$ equal to $9 \mathrm{~mm}$. It is worth noting that the axis of the dipoles are oriented differently: $D_{1}, D_{3}$ and $D_{5}$ vertically along $(O z)$, and horizontally for the two others $D_{2}$ along $(\mathrm{Oy})$ and $\mathrm{D}_{4}$ along $(-\mathrm{Ox})$.

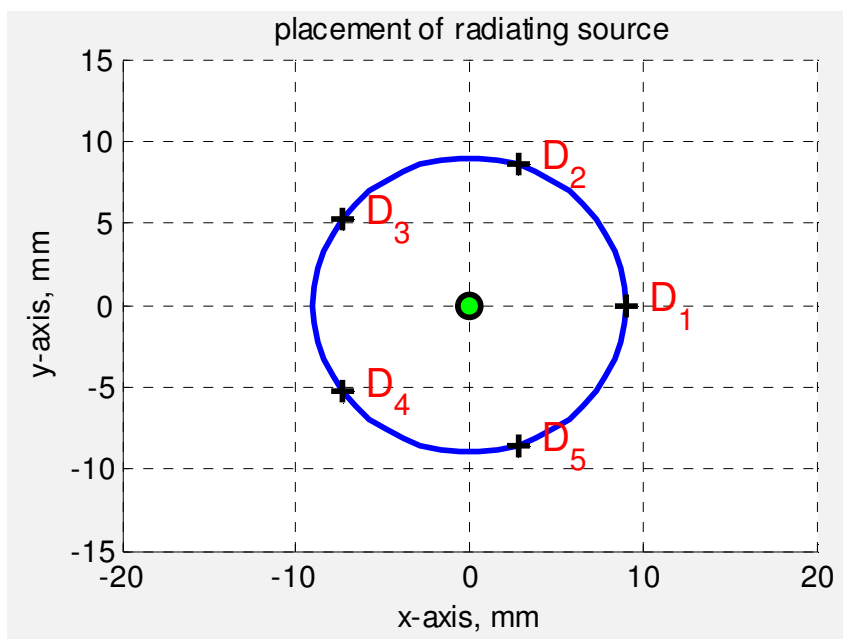

Fig. 5 : Association of five electric dipoles in the horizontal plan $z=0$.

Throughout the calculation and the simulation, the five dipoles sources were simultaneously excited by a baseband pulse current whose the temporal evolution and the frequency spectrum (in semi-log plot) are displayed in Fig. 6. One points out that this current form can be assumed as the undesired perturbations as the eventual harmonics in the electronic systems. One finds that more than $80 \%$ of the injected signal spectrum energy belongs in the baseband frequency delimited to $5 \mathrm{GHz}$.
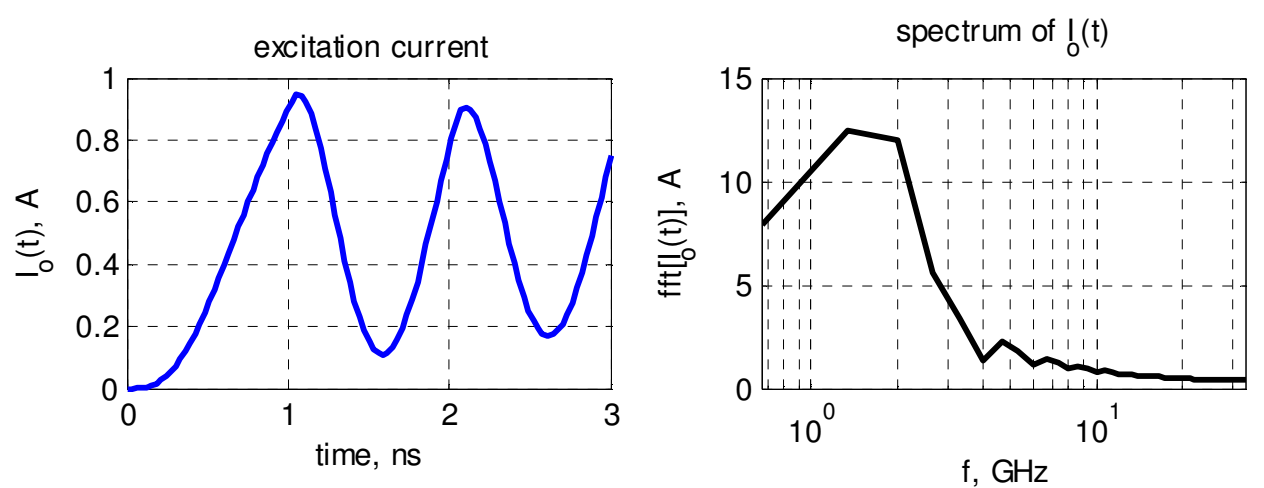

Fig. 6: Temporal plot of the excitation current, $I_{o}(t)$ (in left) and its frequency spectrum,

$$
I_{o}(f)=f f t\left[I_{o}(t)\right] \text { (in right). }
$$

After implementation of the analytical relations presented in section 2 and following succintly the methodology of the proposed calculation technique with Matlab, one obtaines the magnetic field radiations depicted here after. During the computation, a rectangular plane delimited by $x_{\min }=-35 \mathrm{~mm}, x_{\max }=35 \mathrm{~mm}, y_{\min }=-40 \mathrm{~mm}$ and 
$y_{\max }=40 \mathrm{~mm}$ was considered for the calculation of $H_{x}, H_{y}$ and $H_{z}$. With the excitation current shown in Fig. 6, one gets the results represented by the cartographies of Fig. 7 at $t=1 \mathrm{~ns}$ and $z=2 \mathrm{~mm}$.

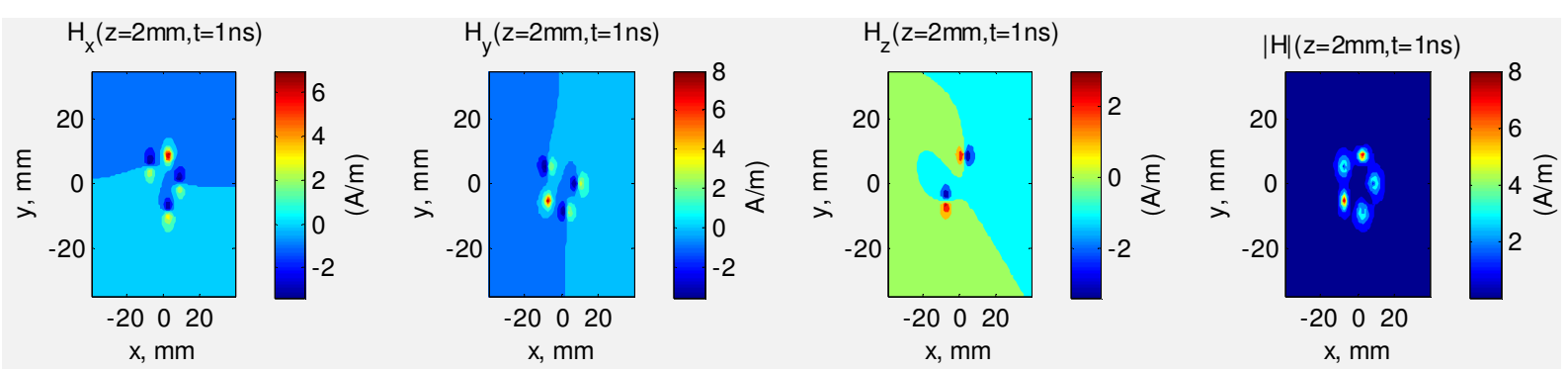

Fig. 7: Cartographies of magnetic field components $H_{x}, H_{y}$ and $H_{z}$, and the whole modulus $|H|$ at the time, $t=1 \mathrm{~ns}$ and calculated in the horizontal plane placed at the height, $z=2 \mathrm{~mm}$ above the plane of the radiating sources.

With the proposed computation process developed in the previous section, one can determine the cartographies at $z_{0}=\{5 \mathrm{~mm}, 10 \mathrm{~mm}, 15 \mathrm{~mm}\}$ from the calculated magnetic field components $H_{x}(t), H_{y}(t)$ and $H_{z}(t)$ at $z=2$ $\mathrm{mm}$. Therefore, one gets the comparative results displayed in Figs. 8. One can see that the presented results are in good correlation with those yielded from the direct calculation.

Moreover, one underlines also that as depicted in Fig. 9 that with the proposed method, one can forecast the global magnitude behaviour $\left|H\left(x, y, z_{0}\right)\right|$ of the transient radiated near-field at certain distances of the given EM field map.

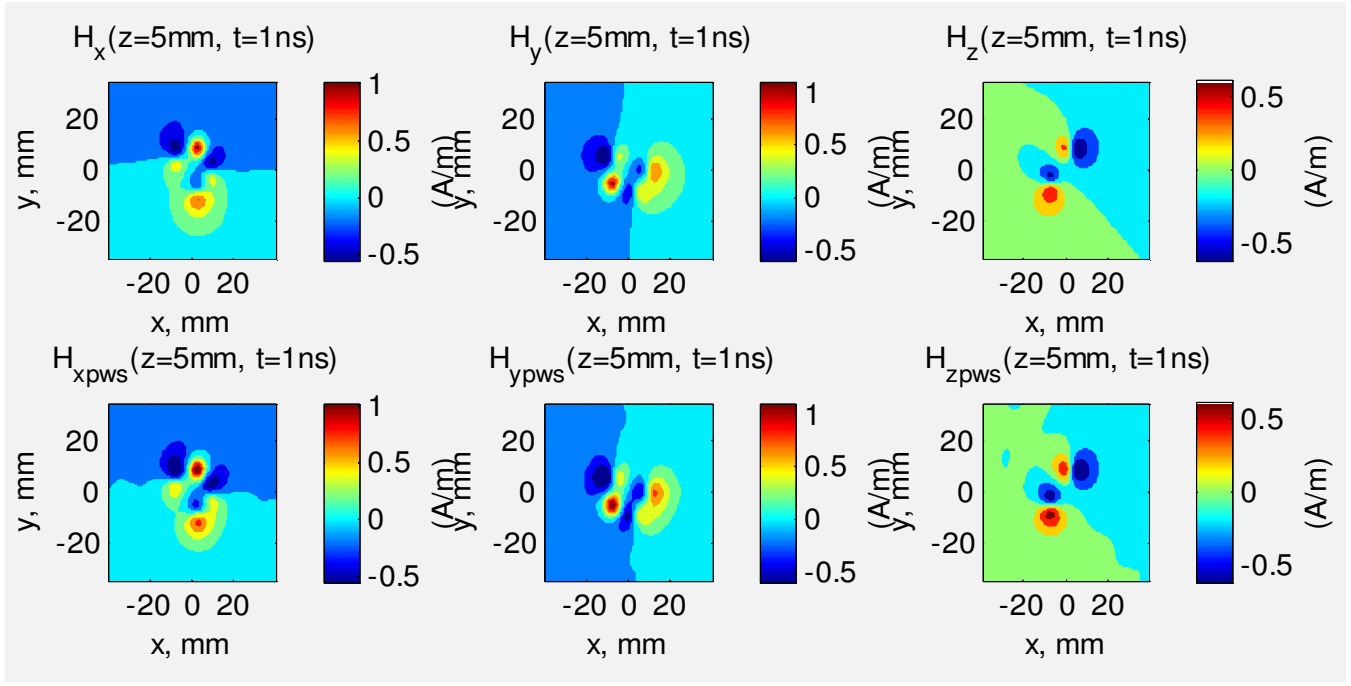

(a) 


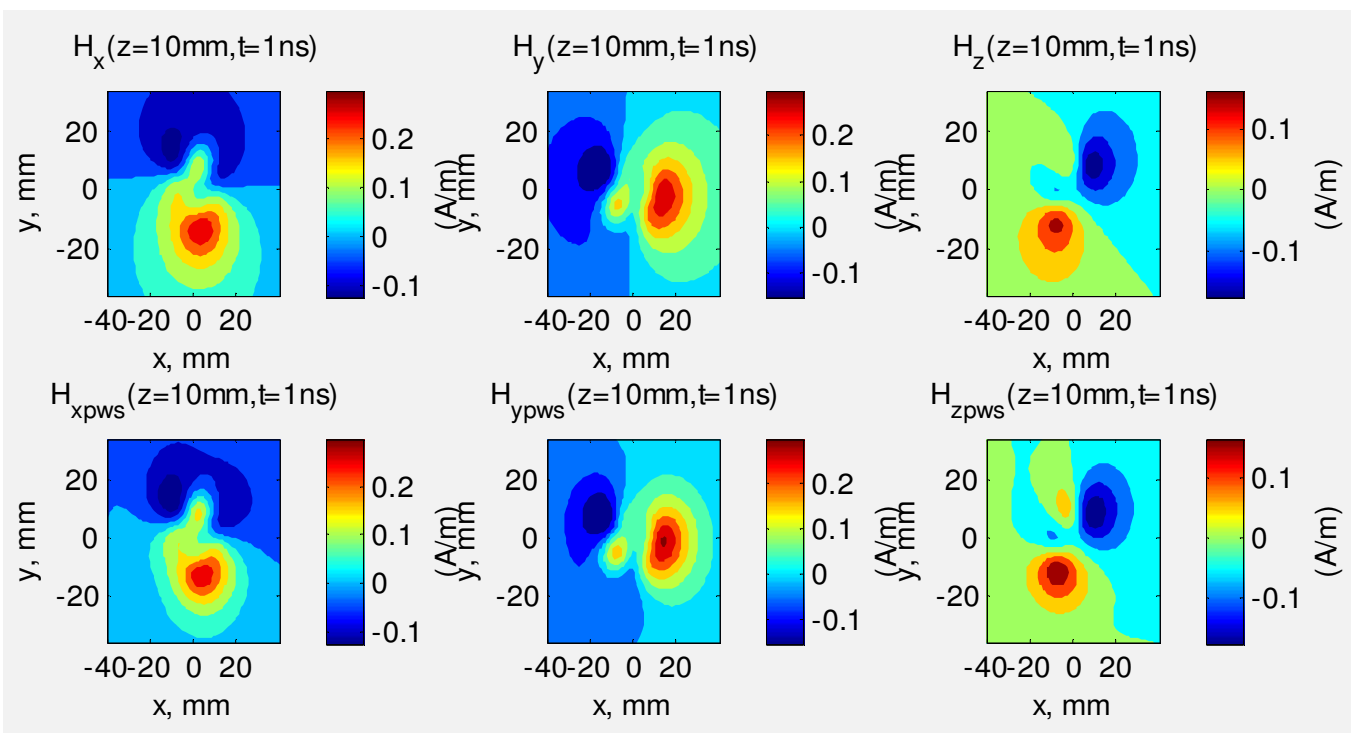

(b)
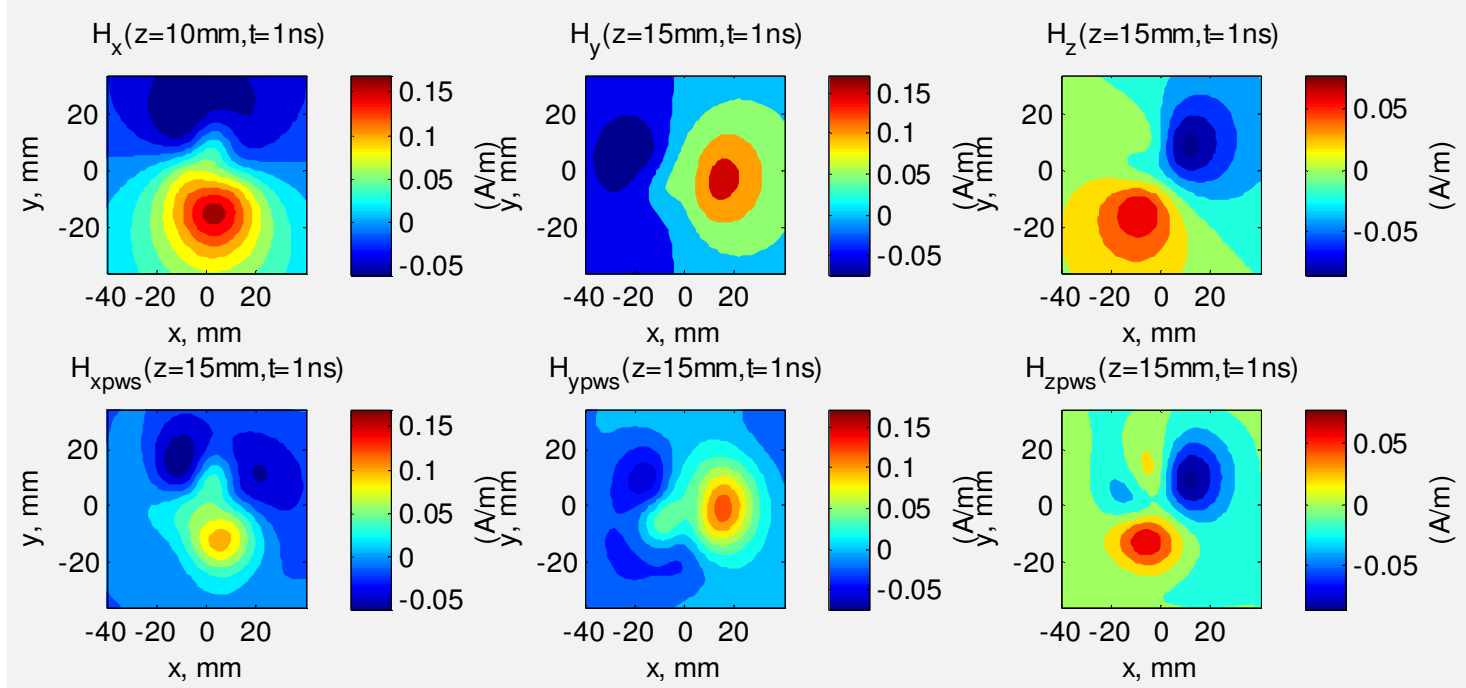

$H_{z p w s}(z=15 \mathrm{~mm}, t=1 \mathrm{~ns})$

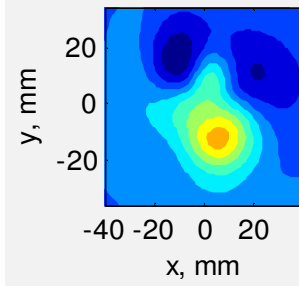

(c)

Fig. 8: Comparison between the cartographies of magnetic field components $H_{x}, H_{y}$ and $H_{z}$ directly calculated and that one extracted from the PWS method at the time, $t=1 \mathrm{~ns}$ and calculated in the horizontal plane placed at the height, (a) $z=5 \mathrm{~mm}$, (b) $z=10 \mathrm{~mm}$ and (c) $z=15 \mathrm{~mm}$ above the plane of the radiating sources.

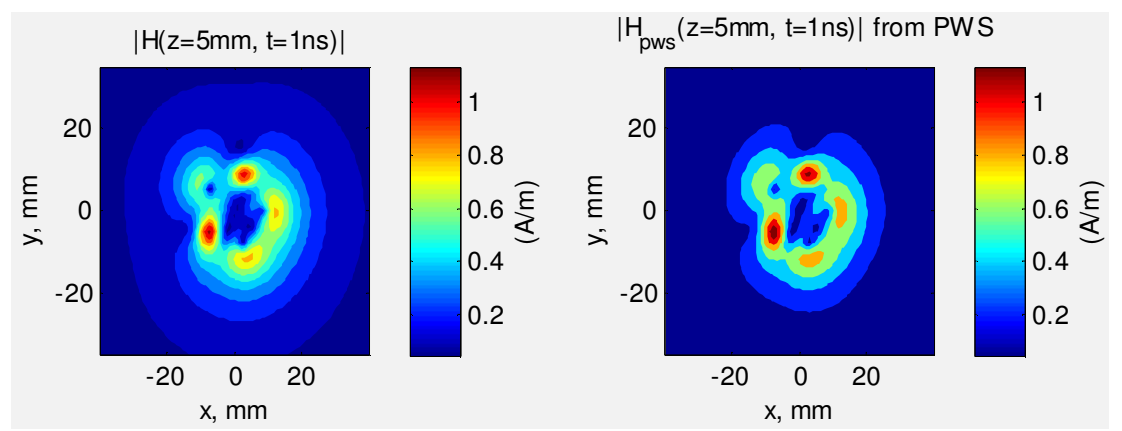

(a) 


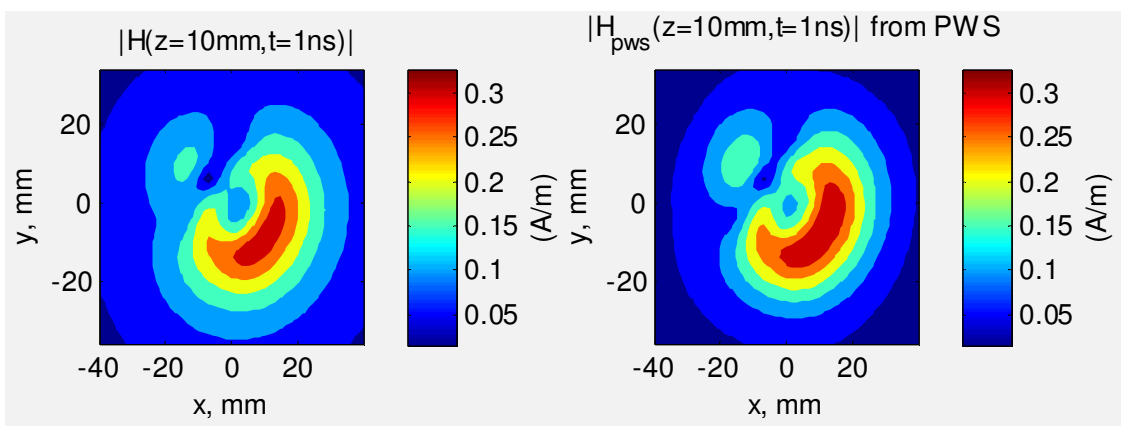

(b)

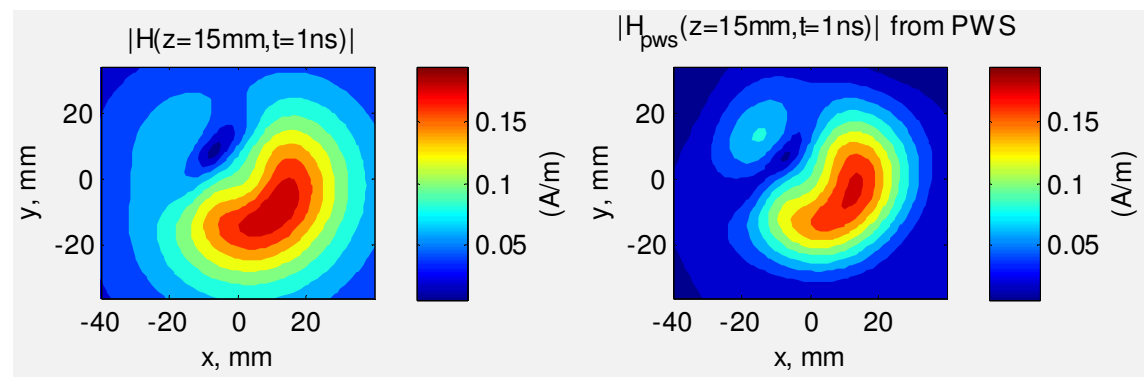

(c)

Fig. 9: Comparison between the cartographies of the global magnetic field modulus $|H|$ directly calculated and that one extracted from the PWS method at the time, $t=1 \mathrm{~ns}$ and calculated in the horizontal plane placed at the height, (a) $z=5 \mathrm{~mm}$, (b) $z=10 \mathrm{~mm}$ and (c) $z=15 \mathrm{~mm}$ above the plane of the radiating sources.

In order to evidence more clearly the efficiency of the herein introduced technique, comparisons of the profiles of $H_{x}, H_{y}$ and $H_{z}$ along $O y$ are also performed. As depicted in Figs. 10, once again, as expected very good agreement with the reference data is observed. It was found also that the error is generally higher when moving away to the radiated sources.

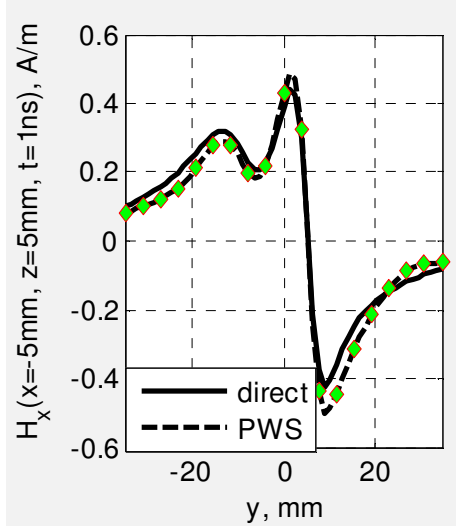

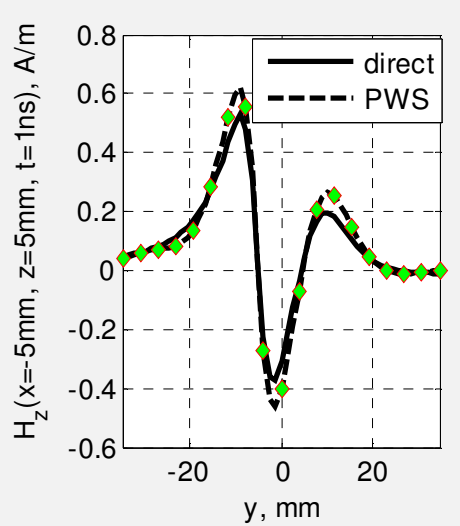

(a) 


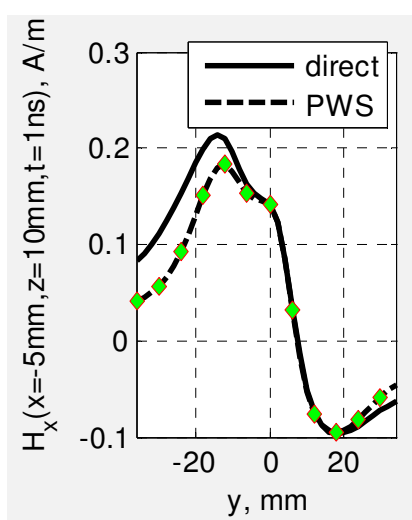

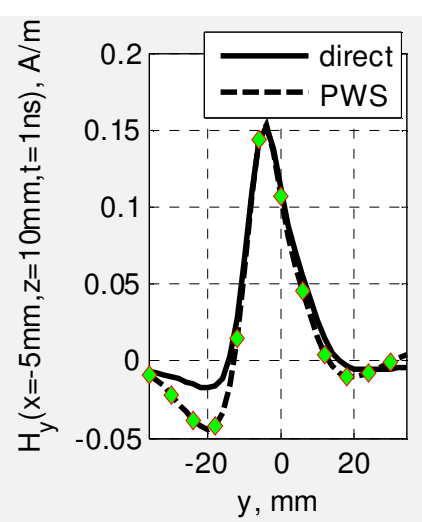

(b)

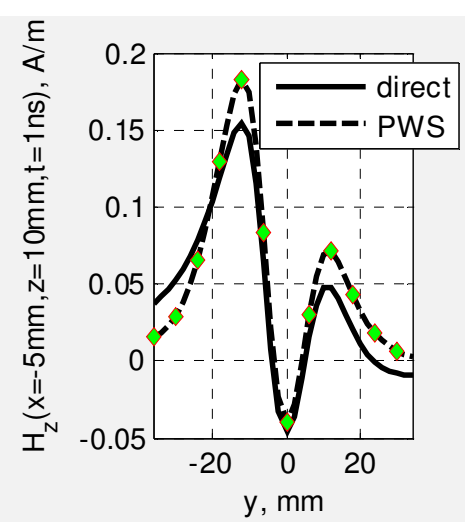

$\mathrm{y}, \mathrm{mm}$

Fig. 10: Profiles of H-field components in the cut-plane with $x=-5 \mathrm{~mm}$ at the height, (a) $z=5 \mathrm{~mm}$ and (b) $z=10 \mathrm{~mm}$ above the plane of the radiating sources.

One points out that with the time-vector containing 101 points and the detection plane resolutions with $N_{x} \mathrm{x} N_{y}=141 \mathrm{x} 141$ points, the Matlab elapsed time during the calculation is 1561 seconds by using Intel Core 2 Duo CPU, P8400 @ $2.26 \mathrm{GHz}$ and with memory 2 Go RAM. The error contributed by the proposed technique is more and more significant when moving away the radiating source in particular if the distance between the computation plane and the reference plane exceeds $\lambda / 15$. To improve these results, higher time and space resolutions should be set. But for this representation, it is interesting to point out that the error can be significant in areas where the field values is 10 times lower than the absolute value of the maximal field value.

\section{CONCLUSION}

A relevant computation method enabling the near-field/near-field transform in time-domain for the RF-/highfrequency EM applications is reported. The presented method permits the reconstitution of the maps of EMnear-field emission at any distance of the given 2D-data according to any transient perturbations. The developed computation process is based on the use of the PWS principle combined to the fft operation.

As reported in [26-28], till now, the PWS theory was generally employed for the prediction of the EM field radiation for example from near- to far-field limited only in frequency domain. The methodology of the proposed technique is described. In order to evidence the functionality of the method, the magnetic field mapped in the plane placed at the height, $z=2 \mathrm{~mm}$ from the reference plane where are located the electric dipoles excited simultaneously by a transient signal presenting spectrum presenting a frequency range more than $5 \mathrm{GHz}$ is considered. Hence, a comparison between the components of the magnetic field directly calculated with the analytical formulation of $H_{x}, H_{y}$ and $H_{z}$ and those extracted from the introduced PWS method at different distances, $\{3 \mathrm{~mm}, 8 \mathrm{~mm}, 13 \mathrm{~mm}\}$ from the given planar magnetic field data was made. Therefore, a good agreement between the proposed computation results and the direct analytical calculations was realized.

In the continuation of this work, we have planned to apply the presented computation technique for the study of transient radiations of electronic devices in particular for the prediction of the undesired infrequent radiation in the electrical and electronic systems where frequency analysis is not enough for the modeling of the disturbing radiated emission.

\section{REFERENCES}


[1] Winter W. and Herbrig M., "Time domain measurement in automotive applications," IEEE Int. Symp. EMC, Austin, Texas, USA, pp. 109-115, Aug. 17-21 2009.

[2] Braun, S. Gülten E., Frech A. and Russer P., “Automated measurement of intermittent signals using a timedomain EMI measurement system,” IEEE Int. Symp. EMC, Austin, Texas, USA, pp. 232-235, Aug. 17-21 2009.

[3] Rioult J., Seetharamdoo D. and Heddebaut M., "Novel electromagnetic field measuring instrument with real-time visualization,” IEEE Int. Symp. EMC, Austin, Texas, USA, pp. 133-138, Aug. 17-21 2009.

[4] Song, Z. Donglin, S. Duval, F. Louis A. and Fei D., "A novel electromagnetic radiated emission Source identification methodology,” Asia-Pacific Symp. EMC, Pekin, China, Apr. 12-16 2010.

[5] Edwards R. S., Marvin A. C. and Porter S. J., "Uncertainty analyses in the finite-difference time-domain method,” IEEE Trans. EMC, Vol. 52, Issue 1, pp. 155-163, Feb. 2010.

[6] Liu L., Cui X. and Qi L., "Simulation of Electromagnetic Transients of the Bus Bar in Substation by the Time-Domain Finite-Element Method,” IEEE Trans. EMC, Vol. 51, Issue 4, pp. 1017-1025, Nov. 2009.

[7] Jauregui R., Riu P. I. and Silva F., “Transient FDTD Simulation Validation,” IEEE Int. Symp. EMC, Fort Lauderdale, Florida, USA, pp. 257-262, Jul. 25-30 2010.

[8] Cicchetti R., "Transient analysis of radiated field from electric dipoles and microstrip lines," IEEE Trans. Ant. Prop., Vol. 39, Issue 7, pp. 910-918, Jul. 1991.

[9] Song J. and Chen K.-M., "Propagation of EM pulses excited by an electric dipole in a conducting medium,” IEEE Tran. Ant. Prop., Vol. 41, No. 10, pp. 1414-1421, 1993.

[10] Lakhtakiaa A., Varadana V. K. and Varadana V. V., "Time-harmonic and time-dependent radiation by bifractal dipole arrays,” Int. J. Electronics, Vol. 63, Issue 6, pp. 819-824, Dec. 1987.

[11] Schantz H. G., "Electromagnetic energy around hertzian dipoles,” IEEE Tran. Ant. Prop. Magazine, Vol. 43, No. 2, pp. 50-62, Apr. 2001.

[12] Jun-Hong W., Lang J. and Shui-Sheng J., "Optimization of the dipole shapes for maximum peak values of the radiating pulse,” IEEE Tran. Ant. Prop. Society Int. Symp., Vol. 1, pp. 526-529, 1997.

[13] Sten J. C.-E. and Hujanen A., "Aspects on the phase delay and phase velocity in the electromagnetic nearfield,” Progress In Electromagnetics Research, Vol. 56, pp. 67-80, 2006.

[14] Shanker, B. Lu M. A., Ergin A. and Michielssen E., "Plane-wave time-domain accelerated radiation boundary kernels for FDTD analysis of 3D electromagnetic phenomena," IEEE Trans. Ant. Prop., Vol. 53, No. 11, pp. 3704-3716, Nov. 2005.

[15] Ravelo B., "E-field extraction from H-near-field in time-domain by using PWS method," PIER B Journal, Vol. 25, pp. 171-189, 2010.

[16] Hertz H. R., "Untersuchungen ueber die Ausbreitung der elektrischen Kraft," (in German) Johann Ambrosius Barth, Leipzig, 1892.

[17] Vives-Gilabert Y., Arcambal C., Louis A., Daran F., Eudeline P. and Mazari B., "Modeling magnetic radiations of electronic circuits using near-field scanning method," IEEE Tran. EMC, Vol. 49, No. 2, pp. 391400, May 2007.

[18] Vives-Gilabert Y., Arcambal C., Louis A., Eudeline P. and Mazari B., "Modeling magnetic emissions combining image processing and an optimization algorithm,” IEEE Tran. EMC, Vol. 51, No. 4, pp. 909-918, Nov. 2009. 
[19] Selin V. I., "Asymptotics of the electromagnetic field generated by a point source in a layered medium," Computational mathematics and mathematical physics, Vol. 41, No. 6, pp. 915-939, 2001.

[20] Kozlov I. P., "Electromagnetic fields in planar-layered media close to a zero of the permittivity," J. Comm. Technol. Electron., Vol. 45, No. 5, pp. 496-502, 2000.

[21] Ebenezer D. D. and Stepanishen P. R., "A wave-vector-time-domain technique to determine the transient acoustic radiation loading on cylindrical vibrators in an inviscid fluid with axial flow," The Journal of the Acoustical Society of America, Vol. 89, No. 1, pp. 39-51, 1991.

[22] Birsan M., "Low-frequency transient (time domain) electromagnetic fields propagating in a marine environment,” Int. J. Numerical Modelling, Devices and Fields, Vol. 17, Issue 3, pp. 325-333, Apr. 2004.

[23] Chew W. C. and Kong J. A., "Electromagnetic field of a dipole on a two-layer earth," Geophysics, Vol. 46, No. 3, pp. 309-315, Mar. 1981.

[24] Smagin S. I. and Mazalov V. N., "Calculation of the electromagnetic fields of dipole sources in layered media,” Doklady Physics, Vol. 50, No. 4, pp. 178-183, Apr. 2005.

[25] Balanis C. A., “Antenna theory: Analysis and design,” 3rd ed., Wiley, New York (USA), 2005.

[26] Paris D. T., Leach W. M. and Joy E. B., "Basic theory of probe-compensated near-field measurements," IEEE Tran. Ant. Prop., Vol. 26, No. 3, pp. 373-379, May 1978.

[27] Wang J. J. H., “An examination of the theory and practices of planar near-field measurement," IEEE Tran. Ant. Prop., Vol. 36, No. 6, pp. 746-753, Jun. 1988.

[28] Leather P. S. H. and Parsons J. D., "Plane wave spectra test-zone fields and simulation of antenna pattern measurements," Electron. Let., Vol. 39, No. 25, pp. 1780-1782, Dec. 2003.

[29] Shi J., Cracraft M. A., Zhang J. and DuBroff R. E., "Using near-field scanning to predict radiated fields," IEEE Ant. Prop. Int. Symp. 1989, San Jose, CA, USA, pp. 1477-1480, Vol. 3.

[30] Higashiyama J. and Tarusawa Y., "Method for measuring radiated electric field excited by linear array antenna using near field to far field transformation,” 18th Int. Zurich Symp. EMC, Munich, Germany, pp. 417420, Sep. 24-28 2007.

[31] Baudry D., Kadi M., Arcambal C., Riah Z., Vives-Gilabert Y., Louis A. and Mazari B., "Plane wave spectrum theory applied to near-field measurements for EMC investigations," Sci. Meas. Technol., IET, Vol. 3, Issue 1, pp. 72-83, Jan. 2009.

[32] Ravelo B., Riah Z., Baudry D. and Mazari B., "E-field extraction from Hx- and Hy- near field values by using plane wave spectrum method," To be published in EPJAP. 